\title{
Design of Multiband Balanced Folded Dipole Antenna Based on a Dual-arm Structure for Mobile Handsets
}

\author{
D. Zhou, R. A. Abd-Alhameed, C. H. See, and P. S. Excell \\ Mobile and Satellite Communications Research Centre, University of Bradford \\ Bradford, West Yorkshire, BD7 1DP, UK
}

\begin{abstract}
In this paper, a balanced antenna for mobile handset applications with enhanced bandwidth performance, that covers four bands (GSM1800, GSM1900, UMTS and 2.4-GHz WLAN), is investigated. The antenna is a slot planar dipole with folded structure and a dual-arm on each monopole. A wide bandwidth planar balun is employed to feed the wideband balanced antenna from an unbalanced source. A prototype of the proposed antenna is fabricated and tested. The performance of the antenna is verified and characterized in terms of return loss, radiation patterns and power gain. The calculated and measured results show good agreement and also confirm good wideband characteristics for the proposed antenna with multiband operation.
\end{abstract}

\section{INTRODUCTION}

Over the recent years, the need to expand the bandwidth of antennas in mobile handheld devices follows from the ever-increasing data rates, and hence spectrum requirements, of mobile devices. The implementation of antenna designs causing minimised coupling with the human head and hand and hence minimised SAR would be attractive to many consumers, thus increasing the market acceptance of devices using such antennas.

A good candidate is a balanced antenna [1]. An antenna with symmetrical structure that is fed with balanced currents to make it electrically symmetrical is said to be a balanced antenna. Dipoles and loops are the most commonly encountered balanced antennas $[2,3]$. In this type of antenna, balanced currents only flow on the antenna element, thus dramatically reducing the effect of current flow on the ground plane. As a result, balanced antennas should have good efficiency and more important to maintain their performance when in use adjacent to the human body.

Narrow band nature for balanced antennas, however, is a limitation for handset applications; even though they were designed and operated in the higher band. Some novel techniques have been proposed for the enhancement of impedance bandwidth for balanced antennas. For example, a genetic algorithm technique has been implemented to improve the impedance bandwidth [4].

In this paper, characteristics of a built-in balanced folded slotted dipole antenna with a novel dual-arm structure for mobile handsets are introduced and analysed in order to realise multipleband operation, including GSM1800, GSM1900, UMTS and WLAN (2.4 GHz). A planar balun with corresponding wide bandwidth for feeding the proposed antenna was implemented in a hardware realization. In the analysis, an electromagnetic simulator based on the finite integration technique (FIT) was applied to calculate return loss and radiation patterns [5].

\section{ANTENNA DESIGN CONCEPT AND STRUCTURE}

The first antenna design attempted was designed in free space and then modifications were introduced to achieve wideband operation for mobile handset applications. The antenna, as shown in Fig. 1(a), is mounted on the top of a rectangular conductor plate $(120 \times 50 \mathrm{~mm})$, which can be regarded as the mobile handset chassis or ground plane of a practical mobile phone.

Planar metal-plate monopole antennas have been investigated in the past as good candidates for ultrawide-band applications [6,7]. A pair of such wideband monopole antennas can be integrated together to configure a dipole and still remain a wide bandwidth feature. Following this principle, a wideband planar dipole antenna in free space can be developed. Moreover, like most balanced antennas, the planar dipole antenna works best in free space and, on the contrary, performs badly when placed in the vicinity of other conductors (e.g., mobile phone ground plane). Thus, in order to mitigate the effects of the ground plane causing degradation of antenna performance, a technique was applied by inserting a slot in each one of the arms of the planar dipole to reduce the effects of the induced current on the ground plane. Consequently, a ground plane in which current is induced may have less mutual effect on the proposed mobile antenna. In this way, the antenna may be able 


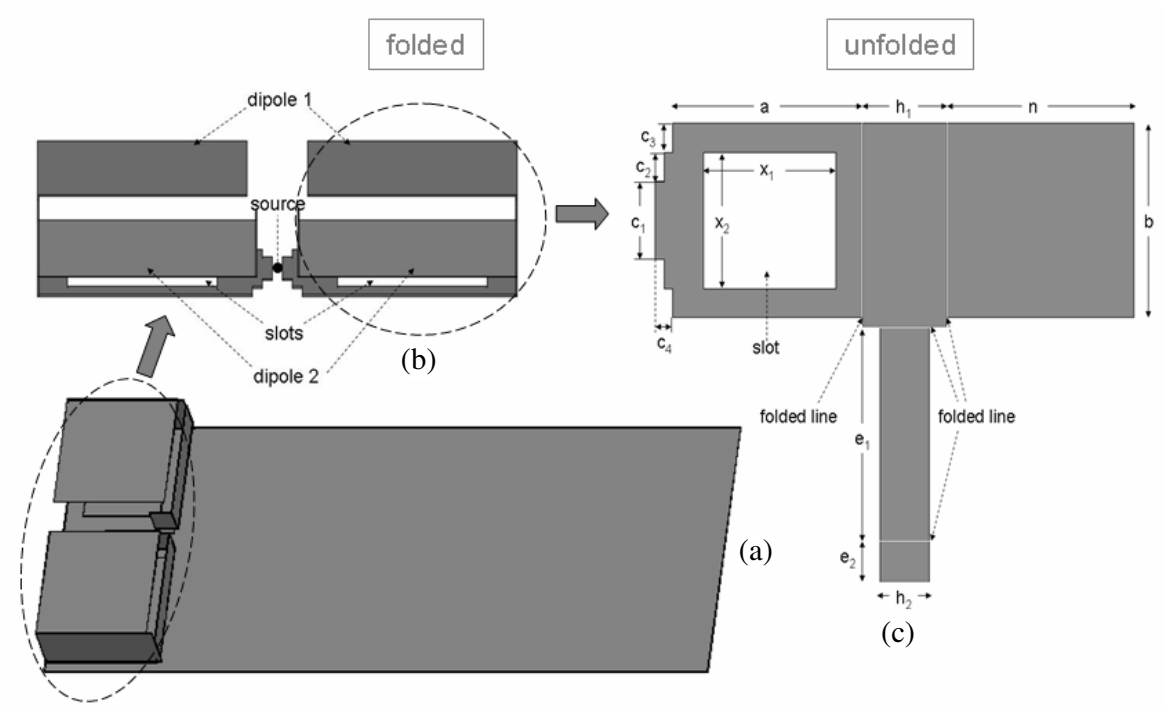

Figure 1: Antenna configuration studied. (a) Balanced folded dipole antenna with conducting plate; (b) Front view of the antenna model; (c) Unfolded structure of the proposed antenna.

to be placed close to the ground plane of the handset (e.g., $1 \mathrm{~mm}$ away). In order to achieve a built-in low profile feature, two arms of dipole antenna were required to be folded.

The foregoing design concept for balanced mobile antennas was applied and implemented in the authors' previous work, as illustrated in [8]. In this work, a further improvement on the impedance bandwidth for the antenna was investigated, in order to cover an additional band at $2.4 \mathrm{GHz}$ for WLAN as a new achievable multiple-band operation, since most mobile phones available in the market have such a feature. Therefore, in order to meet and fulfil the mobile market demands, the proposed balanced mobile antenna was investigated as tailored for multi-band operation.

Initially, an antenna (referring as 'dipole 1' in Fig. 1(b)), operating at around $2 \mathrm{GHz}$, was designed and optimized using the CST simulation package. In addition, a new technique was applied by inserting an additional thin-strip arm (referring as 'dipole 2' in Fig. 1(b)) in one of the arms of the planar dipole to generate another resonant frequency for $2.4 \mathrm{GHz}$ band (see Fig. 1(c)). Using this technique, the single resonant antenna was modified and developed as a wideband dualresonant variant for multi-band operation. It is notable that a symmetrical structure was exploited at the monopole edge near the feed point, as shown in Fig. 1(c), replacing the symmetrical triangle trimming structure as used in [8]. This is because the current configuration has better control on the impedance bandwidth as found in the analysis.

The slot size and location on the antenna, together with the length and location of arm for 'dipole 2 ', including other parameters of the proposed antenna, were adjusted and further optimised to ensure that the design entirely covered the required frequency bands $(1710-2485 \mathrm{MHz})$ at VSWR $\leq 3$.

\section{RESULTS AND DISCUSSION}

For a balanced antenna (e.g., dipole) system, a balun is required as a support feeding network, to provide a balanced feed from an unbalanced source. In this study, a wideband planar balun was adopted [9] and modification was applied to it by rearrangement of the element locations for the purpose of the required practical measurement. Agilent ADS [10] was used to simulate and analyse the characteristic performance of this modified balun. It was found the balun is operated from $1.7 \mathrm{GHz}$ to $3 \mathrm{GHz}$ with maximum $0.9 \mathrm{~dB}$ insertion loss in which the amplitude and phase imbalance are $\pm 0.1 \mathrm{~dB}$ and $\pm 2^{\circ}$, respectively. The balun structure was mounted on Duroid material $\left(\varepsilon_{r}=3.48\right.$, $h=0.8 \mathrm{~mm}$, and $\tan \delta=0.0019$ ).

The optimal antenna configuration studied in terms of return loss, radiation patterns and power gain was found with aid of CST simulator $\left(a=21.5, b=20, h_{1}=9.5, h_{2}=5.5, n=21, c_{1}=8, c_{2}=3\right.$, $c_{3}=3, c_{4}=2, x_{1}=15, x_{2}=14, e_{1}=22$ and $e_{2}=4$; dimensions are in $\mathrm{mm}$ ). For the hardware realisation, copper sheet of thickness $0.15 \mathrm{~mm}$ was used for fabricating the proposed balanced antenna. The ground plane $(0.035 \mathrm{~mm}$ copper thickness $)$ of the proposed antenna was printed on 
one side of the dielectric substrate and planar balun was placed on the other side of the substrate. The non-conductive material was used to suspend the antenna from the ground plane with $1 \mathrm{~mm}$ distance. The position of the two balanced outputs from the balun was carefully located right beneath the feed point of the balanced antenna on the top layer of the substrate. Two thin wires were employed to connect the antenna feed point with the balun through two holes. In this way, the prototype balanced antenna and its feeding network virtually share the same ground plane as

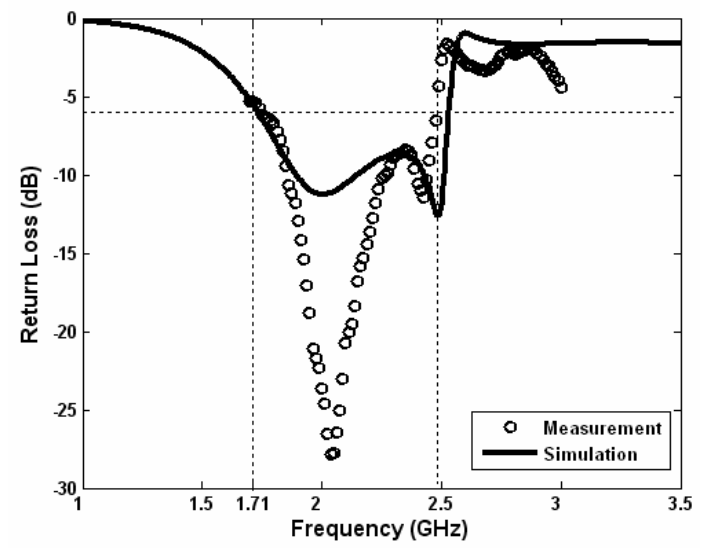

Figure 2: The simulated and measured return loss versus the operating frequency.
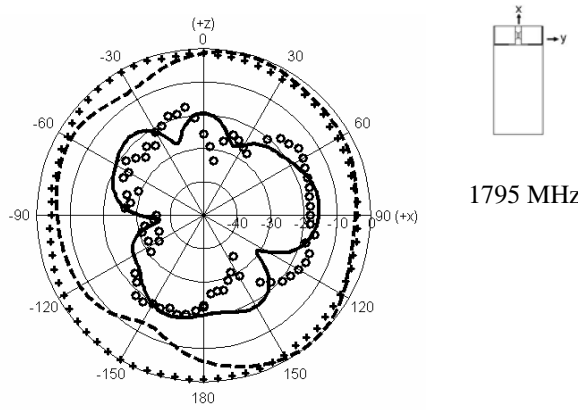

$1795 \mathrm{MHz}$
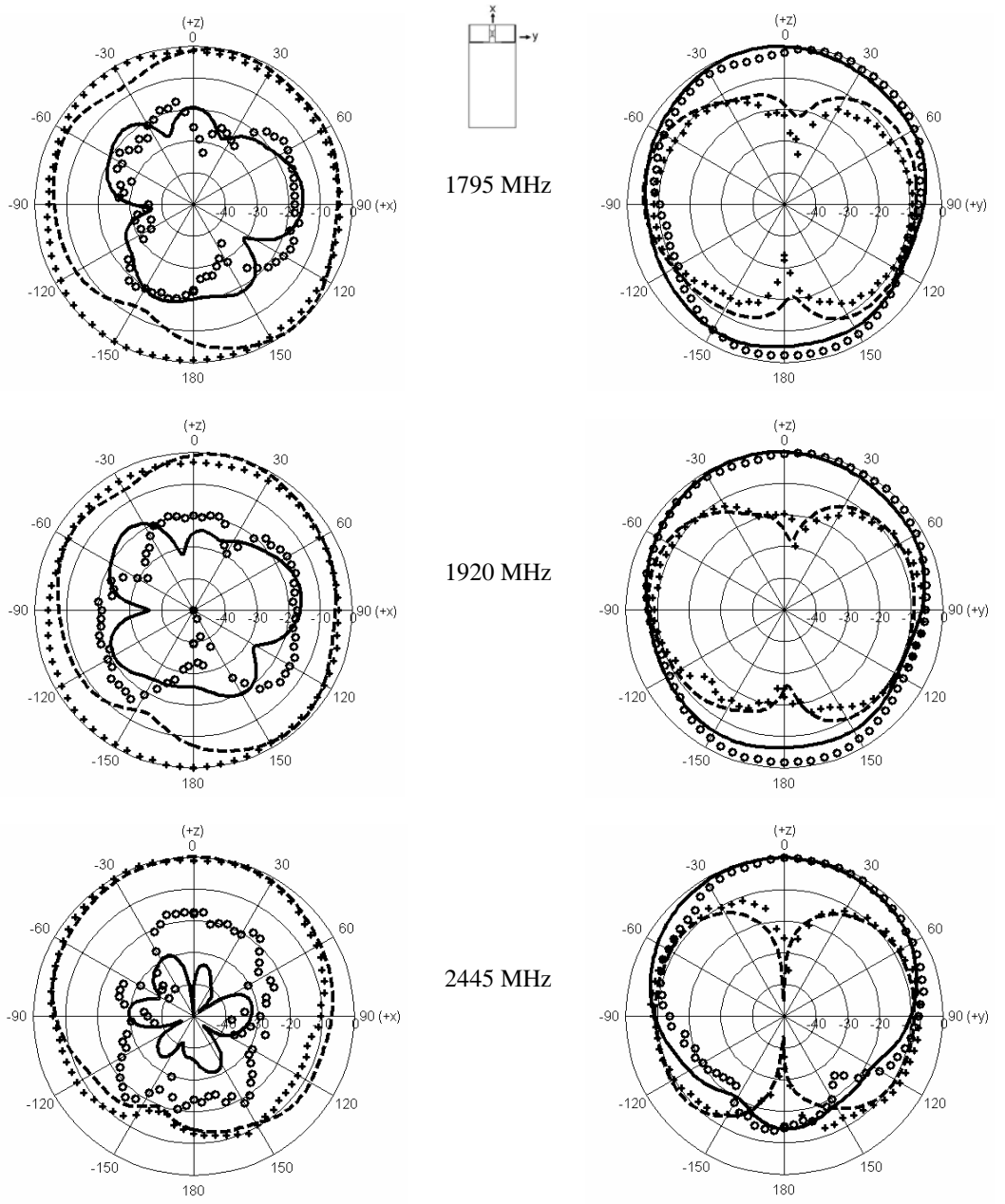

Figure 3: Radiation patterns of the proposed antenna for $1795 \mathrm{MHz}, 1920 \mathrm{MHz}$ and $2445 \mathrm{MHz}$ at: (left) $z x$ plane; (right) zy plane; ('ooo' measured $E_{\theta}$, '—' simulated $E_{\theta}$, '+ ++' measured $E_{\phi}$, and '- - - -' simulated $\left.E_{\phi}\right)$. 
one whole system with close integration.

Figure 2 presents the measured and simulated return loss of the prototype antenna. As can be seen, taking into account the errors caused by manufacturing the proposed antenna, a fairly good agreement between the calculated and measured return loss was observed.

Measurements of the radiation patterns of the prototype were carried out in a far-field anechoic chamber. Two pattern cuts were taken for three selected operating frequencies that cover the designated whole bandwidth in this study. The radiation patterns in the $z x$ plane and $z y$ plane for the balanced folded dipole at 1795, 1920 and $2445 \mathrm{MHz}$ were measured. The results were verified and plotted against the prediction in Fig. 3, in which the patterns of the prototype antenna are seen to be quite similar to each other for the first two cases. Moreover, the $z x$ plane presents a nearly omni-directional radiation pattern in all intended three frequency bands; whereas, the proposed antenna at $2445 \mathrm{MHz}$ tends to radiate at the $+z$ direction in both plane cuts. In this case, the ground plane in this band acts as a good reflector.

Measured broadside antenna power gain for the frequencies across the GSM1800, GSM1900, UMTS and 2.4-GHz WLAN bands were also performed. It is notable that the insertion loss of the feeding network was subsequently compensated for each measured power gain over all bands. It was found that the measured antenna gain was varied between $3.7 \mathrm{~dB}$ and $4.8 \mathrm{~dB}$ over the entire bandwidth considered. For the whole four bands, the peak antenna gain variations are less than $1 \mathrm{dBi}$, as compared with the prediction.

\section{CONCLUSIONS}

A new wideband balanced folded dipole antenna, together with its feeding network (a planar wideband balun), operated over GSM1800, GSM 1900, UMTS and 2.4-GHz WLAN bands, has been presented. The proposed antenna model was experimentally verified in terms the input impedance, radiation pattern and power gain. The simulated and measured results over all frequency bands considered, show a good agreement and this made the proposed antenna an attractive candidate for mobile handset applications.

\section{ACKNOWLEDGMENT}

The authors would like to gratefully acknowledge the support by the Engineering and Physical Sciences Research Council (EPSRC) in the UK under grant EP/E022936.

\section{REFERENCES}

1. Morishita, H., H. Furuuchi, and K. Fujimoto, "Performance of balance-fed antenna system for handsets in vicinity of a human head or hand," IEE Proc.-Microw. Antennas Propag., Vol. 149, No, 2, 85-91, April 2002.

2. Abd-Alhameed, R. A., P. S. Excell, K. Khalil, R. Alias, and J. Mustafa, "SAR and radiation performance of balanced and unbalanced mobile antennas using a hybrid formulation," IEE Proceedings-science, Measurement and Technology Special Issue on Computational Electromagnetics, Vol. 151, No. 6, 440-444, November 2004.

3. Hayashida, S., H. Morishita, Y. Kim, Y. Koyanagi, and K. Fujimoto, "Wideband folded loop antenna for handsets," IEEE AP-S Proc., Vol. 3, 2-5, June 2002.

4. Zhou, D., R. A. Abd-Alhameed, and P. S. Excell, "Bandwidth enhancement of balanced folded loop antenna design for mobile handsets using genetic algorithms," PIERS Online, Vol. 4, No. 1, 136-139, 2008.

5. Computer Simulation Technology Corporation, CST Microwave Studio, Version 5.0, Germany.

6. Ammann, M. J. and Z. N. Chen, "Wideband monopole antennas for multi-band wireless systems," IEEE Antennas and Propagation Magazine, Vol. 45, No. 2, 146-150, April 2003.

7. Wong, K.-L., C.-H. Wu, and S.-W. Su, "Ultrawide-band square planar metal-plate monopole antenna with a trident-shaped feeding strip," IEEE Transaction on Antennas and Propagation, Vol. 53, No. 4, 1262-1269, April 2005.

8. Zhou, D., R. A. Abd-Alhameed, and P. S. Excell, "Wideband balanced folded dipole antenna for mobile handsets," The 2nd European Conference on Antennas and Propagation, No. 39, Session MoPA, Edinburgh, UK, November 11-16, 2007.

9. Zhang, Z. Y., Y. X. Guo, and L. C. Ong, "A new wideband planar balun on a single-layer PCB," IEEE Microwave and Wireless Components Letters, Vol. 15, No. 6, 416-418, June 2005.

10. Advanced Design System, Version ADS2005A, Agilent Technologies. 